\title{
Smart traffic management system with violation detection
}

\author{
Chitra $^{1^{*}}$, J. Vanishree ${ }^{1}$, RaziaSultana $W^{1}$,.Dhawal Kishore Sharma ${ }^{1}$, Pulkit Acharya ${ }^{1}$, Soumya Ranjan ${ }^{1}$ \\ ${ }^{1}$ Vellore Institute of Technology, India, chitra.a@ vit.ac.in
}

\begin{abstract}
This paper proposes a Smart Traffic Management system based on the traffic density present across a four-way crossing using infrared sensors. Along with this, a battery charging circuit is designed to charge a battery using solar energy captured with the help of a solar panel. A Traffic Violation Detection circuit is also proposed which can detect the traffic rule violations by the vehicles during the red light. The circuit is precise, but at the same time simple enough to be implemented in a low-cost processor. The infrared sensors are used to detect the density of the vehicles in a particular lane. Once the traffic density in a lane is detected, the timing of the red and green light is then adjusted according to the data from the sensors. Whenever a traffic light violation is detected by Traffic Violation Detection circuit, the picture of the violator is captured and a SMS alert is generated and sent to the concerned authority.
\end{abstract}

Key words : Infrared sensors, Battery charging circuit, Traffic violation detection.

\section{INTRODUCTION}

Automotive technologies are gaining ground in modern traffic control system since the number of vehicles and passengers are rapidly growing so there is a need for safety critical traffic signal automation. Also, in today's world of energy crisis and global warming conventional sources of energy are not the solution. Alternative sources of energy, especially renewables ones have become vital to handle the problems due to energy crisis [1] [3] [4] [5]. Renewable sources of energy are obtained from naturally replenished sources. This paper explains a battery charging circuit that can charge a battery by harnessing solar energy.

It focuses on the problem of traffic management by creating a smart system which is able to handle the traffic based on the density of vehicles on a particular lane and at the same time it can be supported by solar energy for working [11] - [18].

Modern traffic control systems have three components: Detector, controller and signal display. The detector helps in detecting vehicles in lane and sends the data to the control system which may be automated or a manual system. Controller processes the data and then sends the signal to the output panels. The signal display directs the vehicles on the road whether to pass or stay [2].
The proposed system for traffic management uses IR sensors for detecting the traffic density in a particular lane. Based on the signal from the sensor, the microcontroller changes the duration of green light. The microcontroller used is 8051.To implement the use for green energy, a battery charging circuit is used which can charge a battery by harnessing solar energy using a solar panel [6] -[10]. This circuit operates only when there is a red-light signal for a particular lane. During the red light, vehicles are expected to stop. If someone tries to cross the red signal, then the circuit can detect the violation by using laser and LDR. This circuit requires the violator to break path of the laser which is falling on LDR. Once the path is broken, the LDR sends signal to buzzer which can alert the authority on site.

At the moment of violation, a picture of violator is captured while it is violating the red signal. Along with the picture, an SMS alert is generated and sent to the concerned authority.

The paper is organized as follows. Section 2 deals with the types of traffic management systems and explains the working of them. The control logic of the smart traffic system is explored in Section 3. In Section 4, the methods have been verified and discussed exhaustively. Section 5 concludes this paper.

\section{CONVENTIONAL TRAFFIC MANAGEMENT AND DETECTION SYSTEMS}

\subsection{Types of Traffic Light Systems}

There are different types of traffic management systems. Out of which the most widely employed is discussed in this section.

\subsubsection{Traffic Management System with Fixed Time}

In this method, signals will change in accordance with the preset timings. Signals will continue to repeat the cycle all the time even when there are no vehicles on the signal crossing.

\subsubsection{Adaptive Traffic Light Systems}

In this method, green time is allocated variably. It responds to traffic dynamically based on real time data of the changing traffic patterns (see Figure 1 b) [11]. Signals are generated based on input from vehicle detectors to a controller. The data 


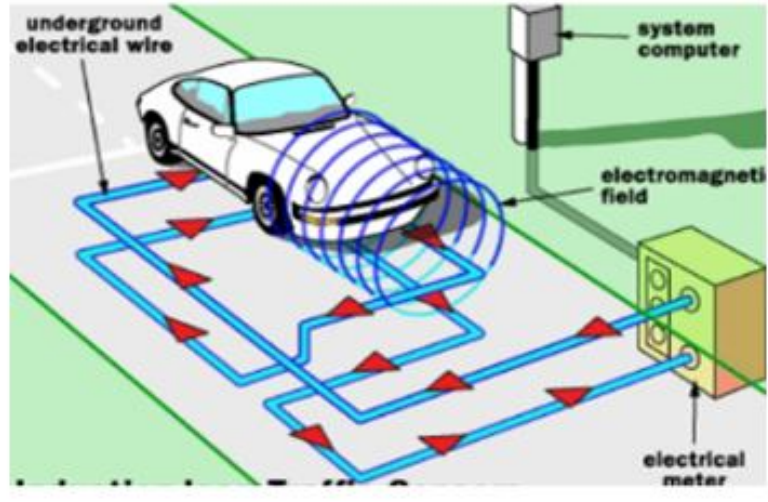

(a)

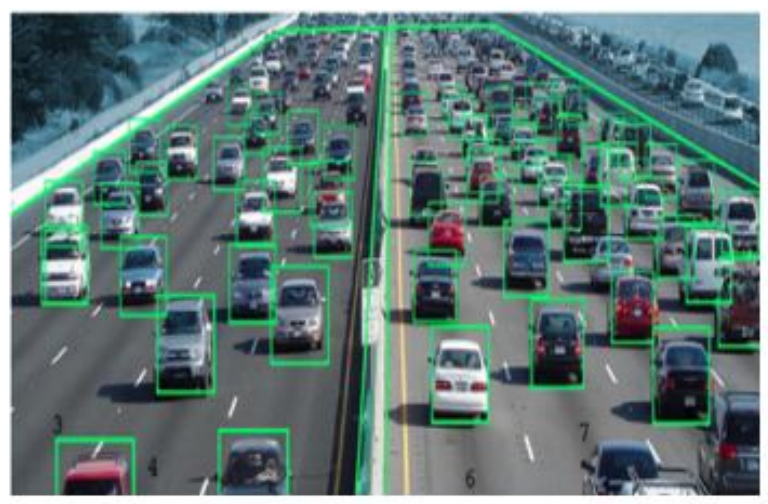

(c)

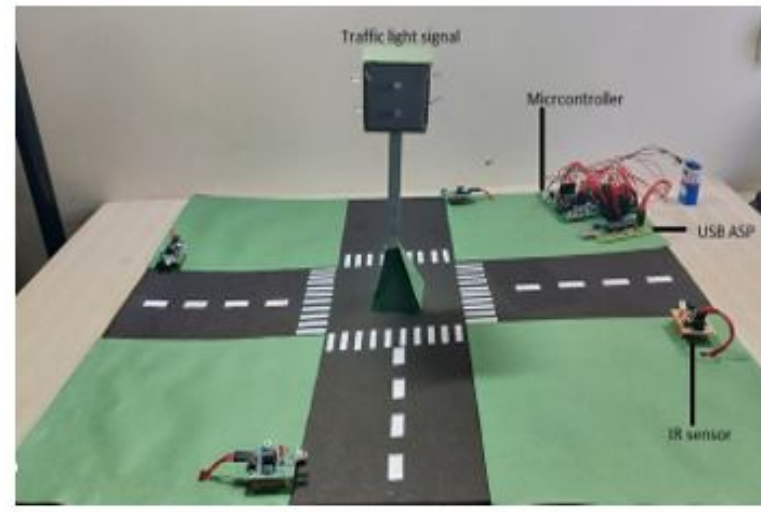

(b)

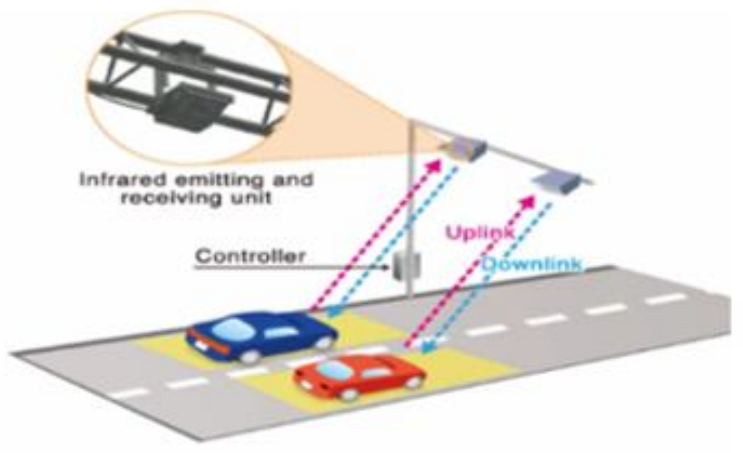

$\Longrightarrow$ (d)

Figure 1. Types of detection systems (a) Induction loop traffic sensor, (b) Prototype of traffic light control system, (c) VideoDetection system and (d) IR detection

collected by the controller is sent to the central processing unit which calculates the density appropriately and allots the timings of the red and green signal based on the calculated density.

\subsection{Types of Detection Systems}

The various detection schemes are mentioned and discussed in this section. The implementation of these systems is discussed in further sections.

\subsubsection{Inductive Loop}

The loop is buried in the traffic lane and is used to generate magnetic field which is powered by the detector. When the vehicles cross the loop, the inductance of the circuit increases. Figure 1a describes this mechanism.

\subsubsection{Video Detection}

Images of moving traffic are captured using the surveillance cameras placed at different positions on the lane, the images are processed rapidly in real time hence providing traffic management effectively [20] - [27]. Figure 1 c shows the mechanism.

\subsubsection{Radio Detection and Ranging}

The IR detectors and optical receivers are installed just above the lanes. They detect vehicles by utilizing reflected waves of near infrared rays. The IR detection system consists of an infrared emitting and receiving unit. In this system, vehicles are detected when they reflect infrared waves back to the receiving unit of the IR detector [19]. These systems have shorter range than the RADAR detection system. Figure $1 \mathrm{~d}$ describes the IR detection mechanism.

\section{PROPOSED SMART TRAFFIC CONTROL LOGIC}

The density of the traffic at a crossing will be sensed with the help of Infrared sensor and then the timings of red and green light will be controlled accordingly to facilitate the ease of movement and to save people's time. To detect the signal breach by any vehicle, a traffic violation detection circuit is incorporated. If a vehicle crosses the line, then the picture of the violator is captured, a SMS alert is generated and sent to the concerned authority.

The components necessary for this circuit are 8051 microcontroller, a digital IR sensor, 8 core cables and connecting wires, red and green LEDs, resistors and a 9v battery and a battery cap. To supply power to the circuits, a $9 \mathrm{~V}$ battery is used. Also, to recharge the battery we have designed a Battery charging circuit which uses solar energy to regenerate power in battery. Thereby we implement a system which is compatible with conventional as well as renewable energy and at the same time solves the real-world problem of Traffic management faced in day to life by reducing time at a signal by adaptive time controlling. 


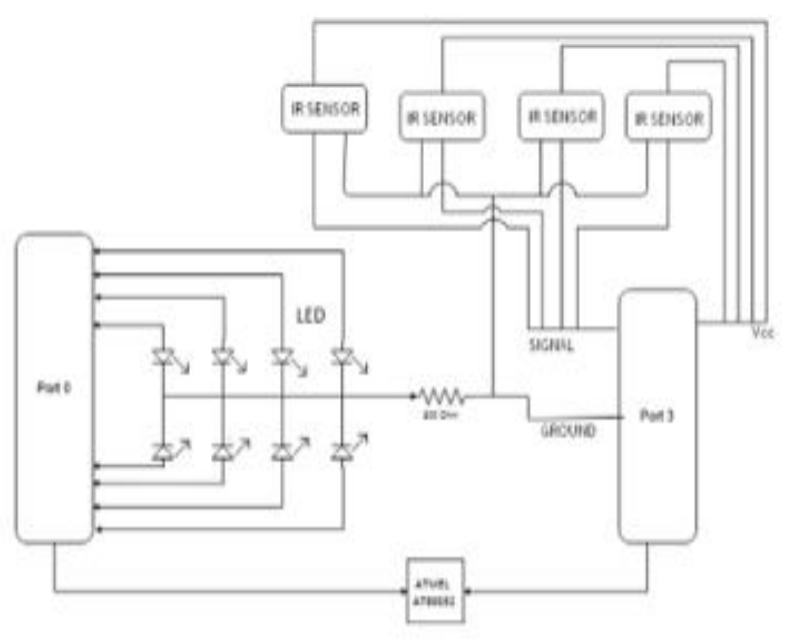

(a)

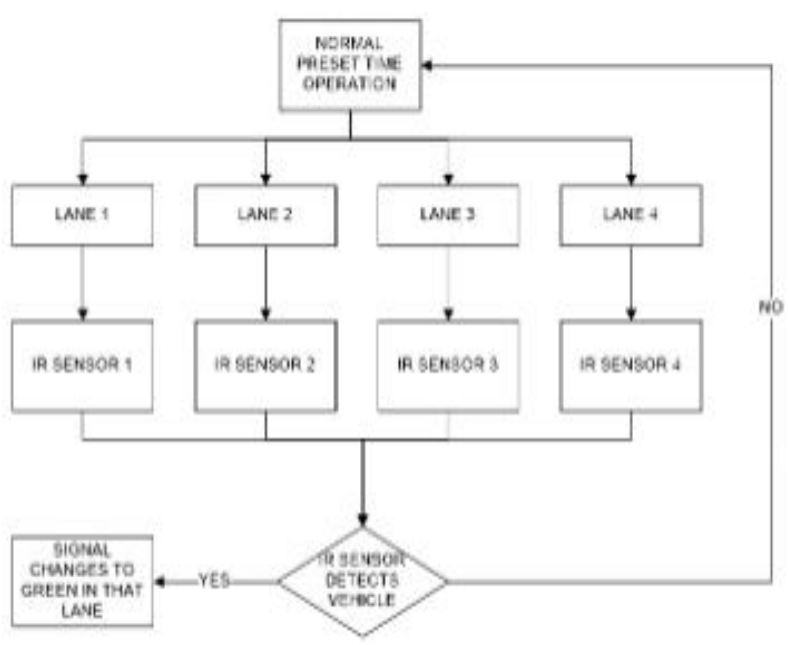

(b)

Figure 2. (a) Traffic light circuit and (b) Circuit and microprocessor flowchart

In the circuit shown in figure 2 a, IR sensors are used to sense the density of traffic at a crossing. The sensors are placed at a location in the lanes where presence of vehicle indicate that it is too crowded. Hence the sensor will detect the vehicle at that location and send the detected signal to the microcontroller.

The microcontroller will then enable the green light of the respective lane. In this way the lane which have maximum traffic will be allotted more time to let the vehicles pass. The flowchart shown in figure $2 \mathrm{~b}$ explains this process in detail.

\subsection{Violation Detection System}

The circuit shown in figure. 3 works in two stages

Stage 1: This circuit uses a Light Depended Resistor. The idea is to switch on or off the buzzer circuit, based on the resistance of the LDR. The resistance and the LDR, connected, between the buzzer and battery are used to form the potential divider network. Transistor is used here for switching purpose. Depending on the voltage across the LDR, the transistor switches ON or switches off the buzzer. If the voltage across the LDR lied above the cut-off value of the voltage $(0.6 \mathrm{~V})$, the transistor gets switched $\mathrm{ON}$ which will turn the buzzer $\mathrm{ON}$ indicating breaking of laser path.

LDR registers low resistance values of the order of $\mathrm{m} \Omega \mathrm{s}$ when it is exposed to the light. The value of the resistance is significantly higher of the order of $\mathrm{M} \Omega$ when the light is not there in the surroundings. A LASER light is projected on the LDR. LASER light is used because it does not deviate from the projected path. When the laser is projected on the LDR, its resistance value is very low. Thus, in accordance with the ohm's law, the voltage across the LDR will also be low which will not be enough to switch $\mathrm{ON}$ the transistor, hence it remains OFF and as a result the buzzer does not operate.

Stage 2: Buzzer operation indicate that the traffic signal is violated by a vehicle. In the stage two, the picture of the vehicle is captured while it is violating the signal and at the same time an SMS alert is generated for the concerned authority.

The operation of buzzer is detected by the arduino. For this, an analog pin and the ground pin are connected in parallel with the buzzer. Once the buzzer operates, the voltage is read in arduino and if the detected voltage crosses a particular threshold, the GSM module is used to generate the

alert SMS. Also, a keyword is sent to python which directs the camera hardware to capture the image of the vehicle while it is violating the traffic signal, as shown in figure 5.

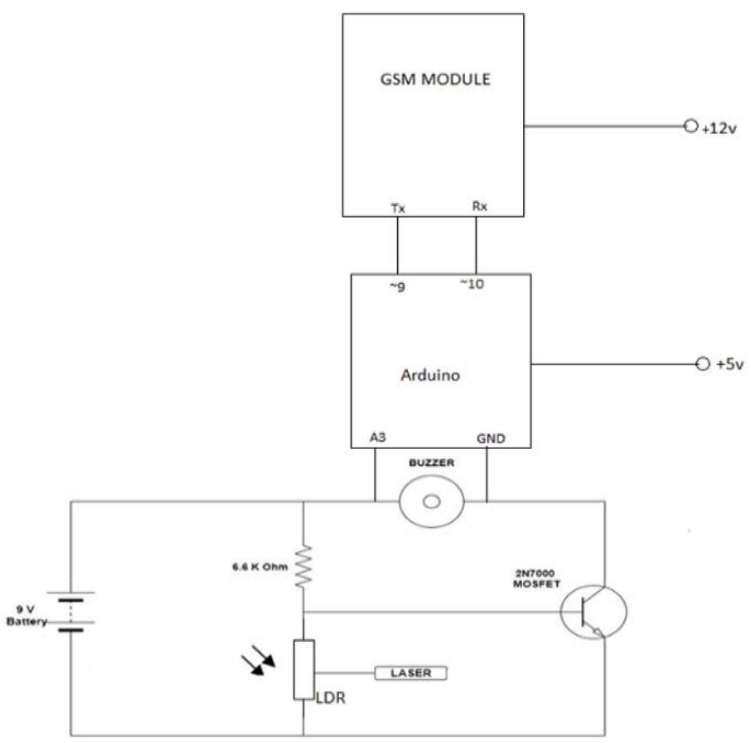

Figure 3. Traffic violation detection circuit

\subsection{Solar Battery Charging Module}

Solar power is harnessed directly through the use of photovoltaic cells that are used for the conversion of heat to electricity. They are made of semiconductor materials that 
exhibit the photovoltaic effect. Solar power can be indirectly harnessed by using the concentration of sunlight to generate heat which is further used to increase the energy of a working fluid. The energized fluid is used to rotate the steam turbine which ultimately generates electrical power. For the concentration of sunlight at a particular point, mirrors and lenses are used.

The battery charging circuit is as illustrated in figure 4. This circuit uses $21 \mathrm{~V}$ solar panel and a variable regulator IC LM317. Charging current from the solar panel passes through the D1 to IC LM317. By varying the adjust pin of the potentiometer, the current and $\mathrm{V}_{\mathrm{o}}$ of the IC can be controlled. Resistor (R3) controls the current flowing from panel to the battery (charging current) and D2 prevents current from battery from slowing in opposite direction. Thus preventing discharge. LED1 is used to indicate the charging of battery. When battery voltage rises above 6.8 Volts, the Zener starts conducting and switches "ON" the transistor BC548 by supplying the base current. The transistor then short circuit the $\mathrm{V}_{\mathrm{o}}$ pin with the ground to stop charging.

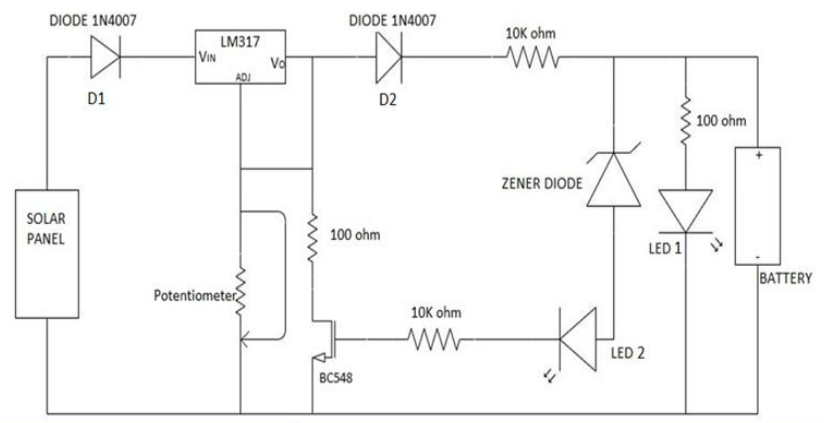

Figure 4. Battery charging circuit

\section{RESULTS AND DISCUSSION}

Traffic density is controlled by using the IR sensor by detecting the presence of vehicle and the correct results are obtained. The battery charging circuit designed in this paper is giving correct results by charging the battery. When the battery is charged then the power from the solar panel cuts off and thus prevent the battery from any damage. This leads to the safety of the battery. The traffic violation detection circuit implemented in this paper helps to catch people who are breaking the rule by crossing the signal. It works by taking a picture of the violator at the instant he crosses the red light as seen in figure 5 and 6 . A Buzzer blows at the site to indicate the traffic police on the road and also it sends a SMS to the concerned authorities to catch the violator at the next signal.

Some of the constraints are the LDR used in the traffic violation detection circuit can get affected by the presence of surrounding light. Even if the density isn't high enough in a particular lane and a vehicle stops in front of the IR sensor, the green light will be activated. Some alternatives are that in place of LDR, IR sensors can be implemented to detect vehicles in the traffic violation detection circuit. Also, A raspberry pi can be used in the place of a computer for the operation of a surveillance camera.

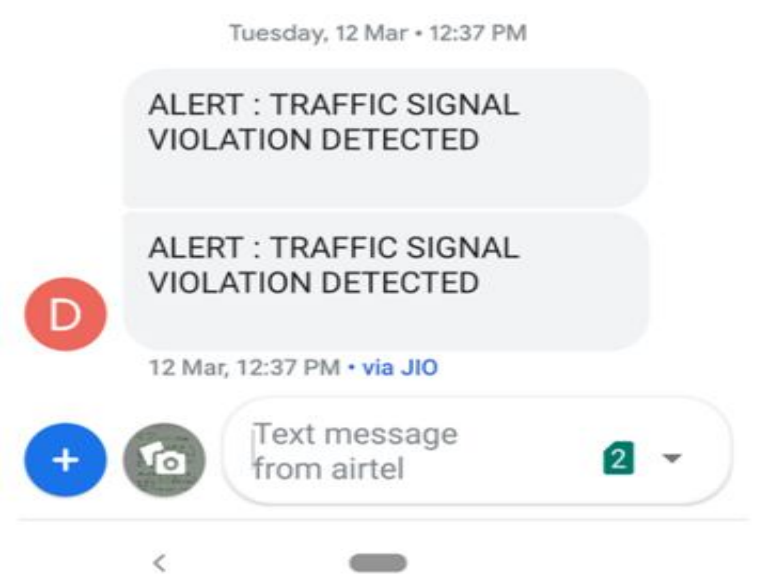

Figure 5. SMS Alert

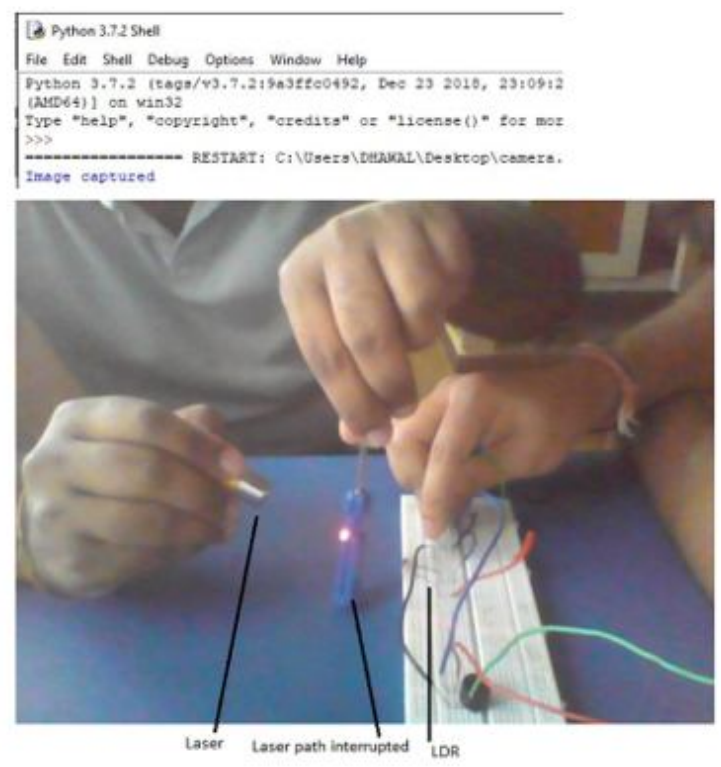

Figure 6. Image captured by camera during violation.

\section{CONCLUSION}

Although the circuit charges the battery connected to it, which is not connected to the main system. This circuit can be integrated with the main circuit with the help of appropriate battery management system. The image of the vehicle is captured while it is violating the traffic signal. Different image processing techniques can be applied to the captured image and vital information (such as the vehicle number) can be extracted. Hence all the results which are required to be obtained from all the circuit are correctly obtained and verified. This system has the capability to be implemented practically for usage and thus real time problems can be solved from it. If all these factors are taken into consideration and implemented with the system than the overall system will give all the needed result with highest accuracy. 


\section{REFERENCES}

[1] Sharma, S., Jain, K. and Sharma, A.. A review on Solar Cells: In Research and Applications. Materials Sciences and Applications, Vol. 6, pp.1145-1155. 2015 Aoyama, K.I., 1994, August. Universal traffic management system (UTMS) in Japan. In Proceedings of VNIS'94-1994 Vehicle Navigation and Information Systems Conference (pp. 619-622). IEEE.

[3] Sierra, C. and Vazquez, A.J., High solar energy concentration with a Fresnel lens. Journal of materials science, 40,Vol.6, pp.1339-1343. 2005.

[4] Gupta, N., Alapatt, G.F., Podila, R., Singh, R. and Poole, K.F.Prospects of nanostructure-based solar cells for manufacturing future generations of photovoltaic modules. International Journal of Photoenergy, 2009.

[5] Bagher, A.M., Vahid, M.M.A. and Mohsen, M., Types of solar cells and application. American Journal of optics and Photonics, 3 .Vol.5, pp.94113. 2015.

[6] Lin, L. T., Huang, H. J., Lin, J. M., \& Young, F. F. A new intelligent traffic control system for Taiwan. In Intelligent Transport Systems Telecommunications,(ITST), (2009) 9th International Conference on (pp. 138-142). IEEE.

[7] Jeon, B.C., Park, W.K., Lee, H.Y., Lee, Y.H. and Lee, S.C. Design \& implementation of a solar powered LED road traffic sign control system. In 2015 International SoC Design Conference (ISOCC) (pp. 321-322). IEEE, November. 2015.

[8] Gaikwad, K. and Lokhande, S., 2015, November. Novel maximum power point tracking (MPPT) algorithm for solar tree application. In International Conference on Energy Systems and Applications (pp. 189-193). IEEE. 2015.

[9] Wang, H., Vinayagam, L., Jiang, H., Cai, Z.Q. and Li, H., 2016, September. New MPPT solar generation implemented with constant-voltage constant-current DC/DC converter. In 51st International Universities Power Engineering Conference (UPEC) (pp. 1-6). IEEE.

[10]Vasant, L.G. and Pawar, V.R., 2017, August. Optimization of solar-wind energy system power for battery charging using MPPT. In 2017 International conference on energy, communication, data analytics and soft computing (ICECDS) (pp. 1308-1310). IEEE..

[11]Leduc G.: Road Traffic Data: Collection Methods and Applications. Working Papers on Energy. Transport and Climate Change, No. 1, Joint Research Centre, Seville, 2008.

[12]Tubaishat, M., Shang, Y. and Shi, H., 2007, January. Adaptive traffic light control with wireless sensor networks. In 2007 4th IEEE Consumer Communications and Networking Conference (pp. 187-191). IEEE.

[13]Al Okaishi, W., Atouf, I. and Benrabh, M., 2019, April. Real-Time Traffic Light Control System Based on Background Updating and Edge
Detection. In 2019 International Conference on Wireless Technologies, Embedded and Intelligent Systems (WITS) (pp. 1-5). IEEE.

[14]Udoakah, Y.N. and Okure, I.G., 2017. Design and implementation of a density-based traffic light control with surveillance system. Nigerian Journal of Technology, 36(4), pp.1239-1248.

[15]Qi, L., Zhou, M. and Luan, W.,. A two-level traffic light control strategy for preventing incidentbased urban traffic congestion. IEEE transactions on intelligent transportation systems, 19(1), pp.1324. 2016.

[16] Knorr, F., Baselt, D., Schreckenberg, M. and Mauve, M., Reducing traffic jams via VANETs. IEEE Transactions on Vehicular Technology,61(8), pp.3490-3498. 2012.

[17]Dash, D.K., India loses Rs 60,000 crore due to traffic congestion: study. The Times of India, New Delhi, 31. 2012.

[18]Baselt, D., Scheuermann, B. and Mauve, M., November. A top-down approach to inter-vehicle communication (poster). In 2011 IEEE Vehicular Networking Conference (VNC) (pp. 206-213). IEEE. 2011.

[19]N Bhatt, A., Rajasekhara Babu, M. and J Bhatt, A.,. Automation testing software that aid in efficiency increase of regression process. Recent Patents on Computer Science, 6(2), pp.107-114. 2013.

[20]Wu, B.F., Kao, C.C., Juang, J.H. and Huang, Y.S., A new approach to video-based traffic surveillance using fuzzy hybrid information inference mechanism. IEEE Transactions on Intelligent Transportation Systems, 14(1), pp.485491. 2012.

[21]Mahalank, S.N., Malagund, K.B. and Banakar, R.M., Device to device interaction analysis in IoT based Smart Traffic Management System: An experimental approach. In 2016 Symposium on Colossal Data Analysis and Networking (CDAN) (pp. 1-6). IEEE.

[22] Javaid, S., Sufian, A., Pervaiz, S. and Tanveer, M., 2018, February. Smart traffic management system using Internet of Things. In 2018 20th International Conference on Advanced Communication Technology (ICACT) (pp. 393-398). IEEE.

[23]Basil, E. and Sawant, S.D., IoT based traffic light control system using Raspberry Pi. In 2017 International Conference on Energy, Communication, Data Analytics and Soft Computing (ICECDS) (pp. 1078-1081). IEEE. August 2017.

[24]W. S. Associate, "Transportation and Economy Report," MDOT State LongRange Transportation Plan, Karachi, 2007.

[25]Rizwan, P., Suresh, K. and Babu, M.R., Real-time smart traffic management system for smart cities by using Internet of Things and big data. In 2016 international conference on emerging technological trends (ICETT) (pp. 1-7). IEEE., October, 2016.

[26] Hegazy Zaher, H. A. Khalifa and Abeer Ahmed, Fuzzy Max Plus Algebra Algorithm for Traffic Problems, International Journal of Emerging Trends in Engineering Research, pp. 530-535, 2019. 
Chitra A et al., International Journal of Emerging Trends in Engineering Research, 8(10), October 2020,7480 - 7485

[27]Alexi Delgado and Leoncio Cueva-Ruiz, Design of a 3D control system using PTV-VISSIM to manage Vehicle traffic, International Journal of Emerging Trends in Engineering Research pp 18191823,2020 